\title{
The Approximate Solutions of Three-Dimensional Diffusion and Wave Equations within Local Fractional Derivative Operator
}

\author{
Hassan Kamil Jassim \\ Department of Mathematics, Faculty of Education for Pure Sciences, University of Thi-Qar, Nasiriyah, Iraq \\ Correspondence should be addressed to Hassan Kamil Jassim; hassan.kamil28@yahoo.com
}

Received 5 June 2016; Accepted 24 August 2016

Academic Editor: Zhenhua Guo

Copyright (C) 2016 Hassan Kamil Jassim. This is an open access article distributed under the Creative Commons Attribution License, which permits unrestricted use, distribution, and reproduction in any medium, provided the original work is properly cited.

\begin{abstract}
We used the local fractional variational iteration transform method (LFVITM) coupled by the local fractional Laplace transform and variational iteration method to solve three-dimensional diffusion and wave equations with local fractional derivative operator. This method has Lagrange multiplier equal to minus one, which makes the calculations more easily. The obtained results show that the presented method is efficient and yields a solution in a closed form. Illustrative examples are included to demonstrate the high accuracy and fast convergence of this new method.
\end{abstract}

\section{Introduction}

The diffusion equation is a partial differential equation that portrays density dynamics in a material that undertakes diffusion. It is also used to describe progression demonstrating diffusive-like performance, for example, the transmission of alleles in a population genetics [1-3]. The three-dimensional diffusion equation in fractal heat transfer involving local fractional derivatives was presented as

$$
\nabla^{2 \alpha} \varphi(x, y, z, t)=\frac{1}{K^{\alpha}} \frac{\partial^{\alpha} \varphi(x, y, z, t)}{\partial t^{\alpha}},
$$

subject to the initial condition

$$
\varphi(x, y, z, 0)=\eta(x, y, z),
$$

where the local fractional Laplace operator is defined as follows (see [4-8]):

$$
\nabla^{2 \alpha}=\frac{\partial^{2 \alpha}}{\partial x^{2 \alpha}}+\frac{\partial^{2 \alpha}}{\partial y^{2 \alpha}}+\frac{\partial^{2 \alpha}}{\partial z^{2 \alpha}} .
$$

$K^{\alpha}$ is a nondifferentiable diffusion coefficient, and $\varphi(x, y, z, t)$ is satisfied with the nondifferentiable temperature distribution, while the three-dimensional wave equation involving local fractional derivatives was presented as

$$
\nabla^{2 \alpha} \varphi(x, y, z, t)=\frac{1}{K^{\alpha}} \frac{\partial^{\alpha} \varphi(x, y, z, t)}{\partial t^{2 \alpha}},
$$

subject to the initial conditions

$$
\begin{aligned}
\varphi(x, y, z, 0) & =\eta_{1}(x, y, z), \\
\frac{\partial^{\partial} \varphi(x, y, z, 0)}{\partial t^{\alpha}} & =\eta_{2}(x, y, z) .
\end{aligned}
$$

Many physical problems are governed by partial differential equations (PDEs), and the solution of these equations has been a subject of many investigators in recent years. The diffusion and wave equations have been successfully modeled for many physical and engineering phenomena such as seismic analysis, rheology, fluid flow, viscous damping, viscoelastic materials, and polymer physics [9-11].

Recently, the diffusion and wave problems were studied by several authors by using local fractional decomposition method [12-15], local fractional variational iteration [1517], local fractional series expansion [18], local fractional functional decomposition method $[19,20]$, local fractional Laplace decomposition method [21], local fractional homotopy perturbation method [22], local fractional similarity solution [23], and local fractional differential transform method $[24,25]$. In this paper, our aims are to present the coupling method of local fractional Laplace transform and variational iteration method, which is called the local fractional variational iteration transform method, and to use 
it to solve three-dimensional diffusion and wave equations with local fractional derivative.

\section{Mathematical Fundamentals}

In this section, we present the basic theory of local fractional calculus and concepts of local fractional Laplace transform (see [12-15]).

Definition 1. One says that a function $f(x)$ is local fractional continuous at $x=x_{0}$; if it holds,

$$
\left|f(x)-f\left(x_{0}\right)\right|<\varepsilon^{\alpha}, \quad 0<\alpha \leq 1
$$

with $\left|x-x_{0}\right|<\delta$, for $\varepsilon, \delta>0$ and $\varepsilon, \delta \in R$. For $x \in(a, b)$, it is called local fractional continuous on $(a, b)$, denoted by $f(x) \in C_{\alpha}(a, b)$.

Definition 2. Setting $f(x) \in C_{\alpha}(a, b)$, the local fractional derivative of $f(x)$ at $x=x_{0}$ is defined as

$$
\begin{aligned}
D_{x}^{\alpha} f\left(x_{0}\right) & =\left.\frac{d^{\alpha}}{d x^{\alpha}} f(x)\right|_{x=x_{0}}=f^{(\alpha)}\left(x_{0}\right) \\
& =\lim _{x \rightarrow x_{0}} \frac{\Delta^{\alpha}\left(f(x)-f\left(x_{0}\right)\right)}{\left(x-x_{0}\right)^{\alpha}}
\end{aligned}
$$

where $\Delta^{\alpha}\left(f(x)-f\left(x_{0}\right)\right) \cong \Gamma(\alpha+1)\left(f(x)-f\left(x_{0}\right)\right)$.

Definition 3. Let one denote a partition of the interval $[a, b]$ as $\left(t_{j}, t_{j+1}\right), j=0, \ldots, N-1$, and $t_{N}=b$ with $\Delta t_{j}=t_{j+1}-t_{j}$ and $\Delta t=\max \left\{\Delta t_{0}, \Delta t_{1}, \ldots\right\}$. The local fractional integral of $f(x)$ in the interval $[a, b]$ is given by

$$
\begin{aligned}
{ }_{a} I_{b}^{(\alpha)} f(x) & =\frac{1}{\Gamma(1+\alpha)} \int_{a}^{b} f(t)(d t)^{\alpha} \\
& =\frac{1}{\Gamma(1+\alpha)} \lim _{\Delta t \rightarrow 0} \sum_{j=0}^{N-1} f\left(t_{j}\right)\left(\Delta t_{j}\right)^{\alpha} .
\end{aligned}
$$

Definition 4. Let $(1 / \Gamma(1+\alpha)) \int_{0}^{\infty}|f(x)|(d x)^{\alpha}<k<\infty$. The Yang-Laplace transform of $f(x)$ is given by

$$
\begin{aligned}
& L_{\alpha}\{f(x)\}= f_{s}^{L, \alpha}(s) \\
&=\frac{1}{\Gamma(1+\alpha)} \int_{0}^{\infty} E_{\alpha}\left(-s^{\alpha} x^{\alpha}\right) f(x)(d x)^{\alpha}, \\
& \quad 0<\alpha \leq 1,
\end{aligned}
$$

where the latter integral converges and $s^{\alpha} \in R^{\alpha}$.

Definition 5. The inverse formula of the Yang-Laplace transforms of $f(x)$ is given by

$$
\begin{aligned}
L_{\alpha}^{-1}\left\{f_{s}^{L, \alpha}(s)\right\}= & f(x) \\
= & \frac{1}{(2 \pi)^{\alpha}} \int_{\beta-i \omega}^{\beta+i \omega} E_{\alpha}\left(s^{\alpha} x^{\alpha}\right) f_{s}^{L, \alpha}(s)(d s)^{\alpha}, \\
& 0<\alpha \leq 1,
\end{aligned}
$$

where $s^{\alpha}=\beta^{\alpha}+i^{\alpha} \omega^{\alpha}$; fractal imaginary unit is $i^{\alpha}$, and $\operatorname{Re}(s)=$ $\beta>0$.

The properties for local fractional Laplace transform used in the paper are given as

$$
\begin{aligned}
L_{\alpha}\{a f(x)+b g(x)\}= & a f_{s}^{L, \alpha}(s)+b g_{s}^{L, \alpha}(s), \\
L_{\alpha}\left\{E_{\alpha}\left(c^{\alpha} x^{\alpha}\right) f(x)\right\}= & f_{s}^{L, \alpha}(s-c) \\
L_{\alpha}\left\{f^{(k \alpha)}(x)\right\}= & s^{k \alpha} f_{s}^{L, \alpha}(s)-s^{(k-1) \alpha} f(0) \\
& -s^{(k-2) \alpha} f^{(\alpha)}(0)-\cdots \\
& -f^{((k-1) \alpha)}(0) \\
L_{\alpha}\left\{E_{\alpha}\left(a^{\alpha} x^{\alpha}\right)\right\}= & \frac{1}{s^{\alpha}-a^{\alpha}} \\
L_{\alpha}\left\{\sin _{\alpha}\left(a^{\alpha} x^{\alpha}\right)\right\}= & \frac{a^{\alpha}}{s^{2 \alpha}+a^{2 \alpha}} \\
L_{\alpha}\left\{x^{k \alpha}\right\}= & \frac{\Gamma(1+k \alpha)}{s^{(k+1) \alpha}} .
\end{aligned}
$$

\section{LFVITM for Three-Dimensional Diffusion Problems}

We first rewrite problem (1) in the local fractional operator form

$$
\begin{aligned}
& L_{t}^{(\alpha)} \varphi(x, y, z, t)=K^{\alpha}\left(L_{x x}^{(2 \alpha)} \varphi(x, y, z, t)\right. \\
& \left.\quad+L_{y y}^{(2 \alpha)} \varphi(x, y, z, t)+L_{z z}^{(2 \alpha)} \varphi(x, y, z, t)\right),
\end{aligned}
$$

where the local fractional differential operators $L_{t}^{(\alpha)}, L_{x x}^{(2 \alpha)}$, $L_{y y}^{(2 \alpha)}$, and $L_{z z}^{(2 \alpha)}$ are defined by

$$
\begin{aligned}
& L_{t}^{(\alpha)}(\cdot)=\frac{\partial^{\alpha}}{\partial t^{\alpha}}(\cdot), \\
& L_{x x}^{(2 \alpha)}(\cdot)=\frac{\partial^{2 \alpha}}{\partial x^{2 \alpha}}(\cdot), \\
& L_{y y}^{(2 \alpha)}(\cdot)=\frac{\partial^{2 \alpha}}{\partial y^{2 \alpha}}(\cdot), \\
& L_{z z}^{(2 \alpha)}(\cdot)=\frac{\partial^{2 \alpha}}{\partial z^{2 \alpha}}(\cdot) .
\end{aligned}
$$

Adopting the local fractional Laplace transform (denoted in this paper by $E_{\alpha}$ ) to both sides of (12) and using the initial condition leads to

$$
\begin{aligned}
& E_{\alpha}\{\varphi(x, y, z, t)\}=\frac{1}{s^{\alpha}} \varphi(x, y, z)+\frac{1}{s^{\alpha}} \\
& \quad \cdot E_{\alpha}\left\{K ^ { \alpha } \left(L_{x x}^{(2 \alpha)} \varphi(x, y, z, t)+L_{y y}^{(2 \alpha)} \varphi(x, y, z, t)\right.\right. \\
& \left.\left.\quad+L_{z z}^{(2 \alpha)} \varphi(x, y, z, t)\right)\right\} .
\end{aligned}
$$


Operating with the inverse of local fractional Laplace transform on both sides of (14) gives

$$
\begin{aligned}
\varphi(x, y, z, t) \\
=\eta(x, y, z) \\
\quad+E_{\alpha}^{-1}\left(\frac{K^{\alpha}}{s^{\alpha}} E_{\alpha}\left\{L_{x x}^{(2 \alpha)} \varphi+L_{y y}^{(2 \alpha)} \varphi+L_{z z}^{(2 \alpha)} \varphi\right\}\right) .
\end{aligned}
$$

$$
\varphi_{n+1}(x, y, z, t)=\varphi_{n}(x, y, z, t)-\frac{1}{\Gamma(1+\alpha)} \int_{0}^{t}\left(L_{\tau}^{(\alpha)} \varphi_{n}-L_{\tau}^{(\alpha)}\left[E_{\alpha}^{-1}\left(\frac{K^{\alpha}}{s^{\alpha}} E_{\alpha}\left\{L_{x x}^{(2 \alpha)} \varphi_{n}+L_{y y}^{(2 \alpha)} \varphi_{n}+L_{z z}^{(2 \alpha)} \varphi_{n}\right\}\right)\right]\right)(d \tau)^{\alpha},
$$

finally, the solution $\varphi(x, y, z, t)$ is given by

$$
\varphi(x, y, z, t)=\lim _{n \rightarrow \infty} \varphi_{n}(x, y, z, t) .
$$

$$
\varphi(x, y, z, 0)=E_{\alpha}\left(x^{\alpha}+y^{\alpha}+z^{\alpha}\right),
$$

$$
K=1 \text {, }
$$

We now consider the initial conditions of (2); namely,

we have

$$
\begin{aligned}
\varphi_{0}(x, y, z, t)= & E_{\alpha}\left(x^{\alpha}+y^{\alpha}+z^{\alpha}\right), \\
\varphi_{n+1}(x, y, z, t)= & \varphi_{n}(x, y, z, t) \\
& -\frac{1}{\Gamma(1+\alpha)} \int_{0}^{t}\left(L_{\tau}^{(\alpha)} \varphi_{n}(\tau)-L_{\tau}^{(\alpha)}\left[E_{\alpha}^{-1}\left(\frac{1^{\alpha}}{s^{\alpha}} E_{\alpha}\left\{L_{x x}^{(2 \alpha)} \varphi_{n}(\tau)+L_{y y}^{(2 \alpha)} \varphi_{n}(\tau)+L_{z z}^{(2 \alpha)} \varphi_{n}(\tau)\right\}\right)\right]\right)(d \tau)^{\alpha} .
\end{aligned}
$$

Consequently, we obtain

$$
\begin{aligned}
\varphi_{0}(x, y, z, t)= & E_{\alpha}\left(x^{\alpha}+y^{\alpha}+z^{\alpha}\right), \\
\varphi_{1}(x, y, z, t)= & \varphi_{0}(x, y, z, t) \\
& -\frac{1}{\Gamma(1+\alpha)} \int_{0}^{t}\left(L_{\tau}^{(\alpha)} \varphi_{0}(\tau)-L_{\tau}^{(\alpha)}\left[E_{\alpha}^{-1}\left(\frac{1^{\alpha}}{s^{\alpha}} E_{\alpha}\left\{L_{x x}^{(2 \alpha)} \varphi_{0}(\tau)+L_{y y}^{(2 \alpha)} \varphi_{0}(\tau)+L_{z z}^{(2 \alpha)} \varphi_{0}(\tau)\right\}\right)\right]\right)(d \tau)^{\alpha} \\
= & E_{\alpha}\left(x^{\alpha}+y^{\alpha}+z^{\alpha}\right)\left(1+\frac{3 t^{\alpha}}{\Gamma(1+\alpha)}\right), \\
\varphi_{2}(x, y, z, t)= & \varphi_{1}(x, y, z, t) \\
& -\frac{1}{\Gamma(1+\alpha)} \int_{0}^{t}\left(L_{\tau}^{(\alpha)} \varphi_{1}(\tau)-L_{\tau}^{(\alpha)}\left[E_{\alpha}^{-1}\left(\frac{1^{\alpha}}{s^{\alpha}} E_{\alpha}\left\{L_{x x}^{(2 \alpha)} \varphi_{1}(\tau)+L_{y y}^{(2 \alpha)} \varphi_{1}(\tau)+L_{z z}^{(2 \alpha)} \varphi_{1}(\tau)\right\}\right)\right]\right)(d \tau)^{\alpha} \\
= & E_{\alpha}\left(x^{\alpha}+y^{\alpha}+z^{\alpha}\right)\left(1+\frac{(3 t)^{\alpha}}{\Gamma(1+\alpha)}+\frac{9 t^{2 \alpha}}{\Gamma(1+2 \alpha)}\right),
\end{aligned}
$$

and so on.

The solution in a nondifferentiable series form

$$
\cdot\left(1+\frac{3 t^{\alpha}}{\Gamma(1+\alpha)}+\frac{9 t^{2 \alpha}}{\Gamma(1+2 \alpha)}+\cdots\right)
$$

$$
\varphi(x, y, z, t)=E_{\alpha}\left(x^{\alpha}+y^{\alpha}+z^{\alpha}\right) \quad \text { is readily obtained. }
$$


Therefore, the exact solution can be written as

$$
\varphi(x, y, z, t)=E_{\alpha}\left(x^{\alpha}+y^{\alpha}+z^{\alpha}+3 t^{\alpha}\right) .
$$

\section{LFVITM for Three-Dimensional Wave Problems}

We first rewrite the problem (4) in the local fractional operator form

$$
\begin{aligned}
& L_{t t}^{(\alpha)} \varphi(x, y, z, t)=K^{\alpha}\left(L_{x x}^{(2 \alpha)} \varphi(x, y, z, t)\right. \\
& \left.\quad+L_{y y}^{(2 \alpha)} \varphi(x, y, z, t)+L_{z z}^{(2 \alpha)} \varphi(x, y, z, t)\right) .
\end{aligned}
$$

Applying the local fractional Laplace transform to both sides of (24) and using the initial condition leads to

$$
\begin{aligned}
& E_{\alpha}\{\varphi(x, y, z, t)\}=\frac{1}{s^{\alpha}} \eta_{1}(x, y, z)+\frac{1}{s^{2 \alpha}} \eta_{2}(x, y, z) \\
& \quad+\frac{1}{s^{2 \alpha}} E_{\alpha}\left\{K ^ { \alpha } \left(L_{x x}^{(2 \alpha)} \varphi(x, y, z, t)\right.\right. \\
& \left.\left.\quad+L_{y y}^{(2 \alpha)} \varphi(x, y, z, t)+L_{z z}^{(2 \alpha)} \varphi(x, y, z, t)\right)\right\} .
\end{aligned}
$$

Operating with the inverse of local fractional Laplace transform on both sides of (25) gives

$$
\begin{aligned}
\varphi(x, y, z, t) \\
=\eta_{1}(x, y, z)+\frac{t^{\alpha}}{\Gamma(1+\alpha)} \eta_{2}(x, y, z) \\
\quad+E_{\alpha}^{-1}\left(\frac{K^{\alpha}}{s^{2 \alpha}} E_{\alpha}\left\{L_{x x}^{(2 \alpha)} \varphi+L_{y y}^{(2 \alpha)} \varphi+L_{z z}^{(2 \alpha)} \varphi\right\}\right) .
\end{aligned}
$$

Deriving both sides of (26) with respect to $t$, we obtain

$$
\begin{aligned}
& L_{t}^{(\alpha)} \varphi(x, y, z, t) \\
& =\eta_{2}(x, y, z) \\
& \quad+L_{t}^{(\alpha)}\left[E_{\alpha}^{-1}\left(\frac{K^{\alpha}}{s^{2 \alpha}} E_{\alpha}\left\{L_{x x}^{(2 \alpha)} \varphi+L_{y y}^{(2 \alpha)} \varphi+L_{z z}^{(2 \alpha)} \varphi\right\}\right)\right] .
\end{aligned}
$$

By the correction function of the irrational method,

$$
\begin{aligned}
& \varphi_{n+1}(x, y, z, t)=\varphi_{n}(x, y, z, t)-\frac{1}{\Gamma(1+\alpha)} \int_{0}^{t}\left(L_{\tau}^{(\alpha)} \varphi_{n}\right. \\
& -\quad L_{\tau}^{(\alpha)}\left[E_{\alpha}^{-1}\left(\frac{K^{\alpha}}{s^{2 \alpha}} E_{\alpha}\left\{L_{x x}^{(2 \alpha)} \varphi_{n}+L_{y y}^{(2 \alpha)} \varphi_{n}+L_{z z}^{(2 \alpha)} \varphi_{n}\right\}\right)\right] \\
& \left.-\eta_{2}(x, y, z)\right)(d \tau)^{\alpha} .
\end{aligned}
$$

Finally, the solution $\varphi(x, y, z, t)$ is given by

$$
\varphi(x, y, z, t)=\lim _{n \rightarrow \infty} \varphi_{n}(x, y, z, t) .
$$

We now consider the initial conditions of (5); namely,

$$
\begin{aligned}
\varphi(x, y, z, 0) & =0, \\
\frac{\partial^{\alpha} \varphi(x, y, z, 0)}{\partial t^{\alpha}} & =3 \sin _{\alpha}\left(x^{\alpha}\right) \sin _{\alpha}\left(y^{\alpha}\right) \sin _{\alpha}\left(z^{\alpha}\right), \\
K & =3 .
\end{aligned}
$$

Starting with the zeroth approximation,

$$
\begin{aligned}
\varphi_{0} & (x, y, z, t) \\
& =\frac{3 t^{\alpha}}{\Gamma(1+\alpha)} \sin _{\alpha}\left(x^{\alpha}\right) \sin _{\alpha}\left(y^{\alpha}\right) \sin _{\alpha}\left(z^{\alpha}\right) .
\end{aligned}
$$

Substituting (31) in (28) we obtain the following successive approximations:

$$
\begin{aligned}
& \varphi_{1}(x, y, z, t)=\varphi_{0}(x, y, z, t)-\frac{1}{\Gamma(1+\alpha)} \int_{0}^{t}\left(L_{\tau}^{(\alpha)} \varphi_{0}(\tau)\right. \\
& -L_{\tau}^{(\alpha)}\left[E_{\alpha}^{-1}\left(\frac{3^{\alpha}}{s^{2 \alpha}} E_{\alpha}\left\{L_{x x}^{(2 \alpha)} \varphi_{0}(\tau)+L_{y y}^{(2 \alpha)} \varphi_{0}(\tau)+L_{z z}^{(2 \alpha)} \varphi_{0}(\tau)\right\}\right)\right] \\
& \left.-3 \sin _{\alpha}\left(x^{\alpha}\right) \sin _{\alpha}\left(y^{\alpha}\right) \sin _{\alpha}\left(z^{\alpha}\right)\right)(d \tau)^{\alpha}=\sin _{\alpha}\left(x^{\alpha}\right) \sin _{\alpha}\left(y^{\alpha}\right) \\
& \cdot \sin _{\alpha}\left(z^{\alpha}\right)\left(\frac{3 t^{\alpha}}{\Gamma(1+\alpha)}-\frac{27 t^{3 \alpha}}{\Gamma(1+3 \alpha)}\right), \\
& \varphi_{2}(x, y, z, t)=\varphi_{1}(x, y, z, t)-\frac{1}{\Gamma(1+\alpha)} \int_{0}^{t}\left(L_{\tau}^{(\alpha)} \varphi_{1}(\tau)\right. \\
& -L_{\tau}^{(\alpha)}\left[E_{\alpha}^{-1}\left(\frac{3^{\alpha}}{s^{2 \alpha}} E_{\alpha}\left\{L_{x x}^{(2 \alpha)} \varphi_{1}(\tau)+L_{y y}^{(2 \alpha)} \varphi_{1}(\tau)+L_{z z}^{(2 \alpha)} \varphi_{1}(\tau)\right\}\right)\right] \\
& \left.-3 \sin _{\alpha}\left(x^{\alpha}\right) \sin _{\alpha}\left(y^{\alpha}\right) \sin _{\alpha}\left(z^{\alpha}\right)\right)(d \tau)^{\alpha}=\sin _{\alpha}\left(x^{\alpha}\right) \sin _{\alpha}\left(y^{\alpha}\right) \\
& \cdot \sin _{\alpha}\left(z^{\alpha}\right)\left(\frac{3 t^{\alpha}}{\Gamma(1+\alpha)}-\frac{27 t^{3 \alpha}}{\Gamma(1+3 \alpha)}+\frac{243 t^{5 \alpha}}{\Gamma(1+5 \alpha)}\right),
\end{aligned}
$$

and so on.

The solution in a nondifferentiable series form

$$
\begin{aligned}
& \varphi(x, y, z, t)=\sin _{\alpha}\left(x^{\alpha}\right) \sin _{\alpha}\left(y^{\alpha}\right) \sin _{\alpha}\left(z^{\alpha}\right) \\
& \cdot\left(\frac{3 t^{\alpha}}{\Gamma(1+\alpha)}-\frac{27 t^{3 \alpha}}{\Gamma(1+3 \alpha)}+\frac{243 t^{5 \alpha}}{\Gamma(1+5 \alpha)} \cdots\right)
\end{aligned}
$$

is readily obtained.

Therefore, the exact solution can be written as

$$
\begin{aligned}
& \varphi(x, y, z, t) \\
& \quad=\sin _{\alpha}\left(x^{\alpha}\right) \sin _{\alpha}\left(y^{\alpha}\right) \sin _{\alpha}\left(z^{\alpha}\right) \sin _{\alpha}\left(3 t^{\alpha}\right) .
\end{aligned}
$$

\section{Conclusion}

In this work, we studied the local fractional variational iteration transform method to solve three-dimensional diffusion and wave equations involving local fractional derivative operator and their nondifferentiable solutions were obtained. This method can also be applied to a large class of system of partial differential equations with approximations that converges rapidly to accurate solutions. 


\section{Competing Interests}

The author declares that there are no competing interests regarding this paper.

\section{Acknowledgments}

Hassan Kamil Jassim acknowledges Ministry of Higher Education and Scientific Research in Iraq for its support of this work.

\section{References}

[1] A. Kilbas, H. M. Srivastava, and J. J. Trujillo, Theory and Applications of Fractional Differential Equations, Elsevier, 2006.

[2] B. Q. Li, Discontinuous Finite Elements in Fluid Dynamics and Heat Transfer, Computational Fluid and Solid Mechanics, Springer, London, UK, 2006.

[3] H. Versteeg and W. Malalasekera, An Introduction to Computational Fluid Dynamics, Prentice Hall, Upper Saddle River, NJ, USA, 2007.

[4] X. J. Yang, Local Fractional Functional Analysis and Its Applications, Asian Academic, Hong Kong, 2011.

[5] X. J. Yang, Advanced Local Fractional Calculus and Its Applications, World Scientific, New York, NY, USA, 2012.

[6] V. Christianto and B. Rahul, "A derivation of proca equations on cantor sets: a local fractional approach," Bulletin of Mathematical Sciences \& Applications, vol. 10, pp. 48-56, 2014.

[7] H.-Y. Liu, J.-H. He, and Z.-B. Li, "Fractional calculus for nanoscale flow and heat transfer," International Journal of Numerical Methods for Heat \& Fluid Flow, vol. 24, no. 6, pp. 1227-1250, 2014.

[8] Y.-J. Hao, H. M. Srivastava, H. Jafari, and X.-J. Yang, "Helmholtz and diffusion equations associated with local fractional derivative operators involving the Cantorian and Cantor-type cylindrical coordinates," Advances in Mathematical Physics, vol. 2013, Article ID 754248, 5 pages, 2013.

[9] A. Carpinteri and F. Mainardi, Fractals and Fractional Calculus in Continuum Mechanics, Springer, New York, NY, USA, 1997.

[10] A. M. Spasic and M. P. Lazarevic, "Electroviscoelasticity of liquid/liquid interfaces: fractional-order model," Journal of Colloid and Interface Science, vol. 282, no. 1, pp. 223-230, 2005.

[11] I. Podlubny, Fractional Differential Equations, vol. 198 of Mathematics in Science and Engineering, Academic Press, New York, NY, USA, 1999.

[12] X. J. Yang, D. Baleanu, and W. P. Zhong, "Approximation solutions for diffusion equation on Cantor time-space," Proceeding of the Romanian Academy A, vol. 14, no. 2, pp. 127-133, 2013.

[13] Z.-P. Fan, H. K. Jassim, R. K. Raina, and X.-J. Yang, "Adomian decomposition method for three-dimensional diffusion model in fractal heat transfer involving local fractional derivatives," Thermal Science, vol. 19, supplement 1, pp. S137-S141, 2015.

[14] H. Jafari and H. K. Jassim, "Local fractional adomian decomposition method for solving two dimensional heat conduction equations within local fractional operators," Journal of Advance in Mathematics, vol. 9, no. 4, pp. 2574-2582, 2014.

[15] D. Baleanu, J. A. T. Machado, C. Cattani, M. C. Baleanu, and X.-J. Yang, "Local fractional variational iteration and decomposition methods for wave equation on Cantor sets within local fractional operators," Abstract and Applied Analysis, vol. 2014, Article ID 535048, 6 pages, 2014.
[16] X.-J. Yang, D. Baleanu, Y. Khan, and S. T. Mohyud-Din, "Local fractional variational iteration method for diffusion and wave equations on Cantor sets," Romanian Journal of Physics, vol. 59, no. 1-2, pp. 36-48, 2014.

[17] S. Xu, X. Ling, Y. Zhao, and H. K. Jassim, "A novel schedule for solving the two-dimensional diffusion problem in fractal heat transfer," Thermal Science, vol. 19, supplement 1, pp. S99-S103, 2015.

[18] A.-M. Yang, X.-J. Yang, and Z.-B. Li, "Local fractional series expansion method for solving wave and diffusion equations on Cantor sets," Abstract and Applied Analysis, vol. 2013, Article ID 351057, 5 pages, 2013.

[19] Y. Cao, W. G. Ma, and L. C. Ma, "Local fractional functional method for solving diffusion equations on Cantor sets," Abstract and Applied Analysis, vol. 2014, Article ID 803693, 6 pages, 2014.

[20] S.-Q. Wang, Y.-J. Yang, and H. K. Jassim, "Local fractional function decomposition method for solving inhomogeneous wave equations with local fractional derivative," Abstract and Applied Analysis, vol. 2014, Article ID 176395, 7 pages, 2014.

[21] H. K. Jassim, "Local fractional Laplace decomposition method for nonhomogeneous heat equations arising in fractal heat flow with local fractional derivative," International Journal of Advances in Applied Mathematics and Mechanics, vol. 2, no. 4, pp. 1-7, 2015.

[22] Y. Zhang, C. Cattani, and X.-J. Yang, "Local fractional homotopy perturbation method for solving non-homogeneous heat conduction equations in fractal domains," Entropy, vol. 17, no. 10, pp. 6753-6764, 2015.

[23] X.-J. Yang, D. Baleanu, and H. M. Srivastava, "Local fractional similarity solution for the diffusion equation defined on Cantor sets," Applied Mathematics Letters, vol. 47, pp. 54-60, 2015.

[24] X.-J. Yang, J. A. T. Machado, and H. M. Srivastava, "A new numerical technique for solving the local fractional diffusion equation: two-dimensional extended differential transform approach," Applied Mathematics and Computation, vol. 274, pp. 143-151, 2016.

[25] H. Jafari, H. K. Jassim, F. Tchier, and D. Baleanu, "On the approximate solutions of local fractional differential equations with local fractional operator," Entropy, vol. 18, no. 150, pp. 1-12, 2016. 


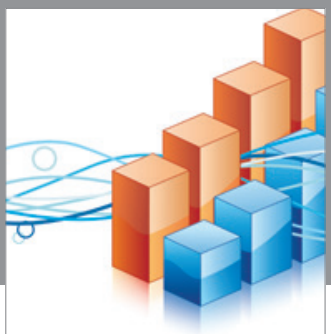

Advances in

Operations Research

vatem alat4

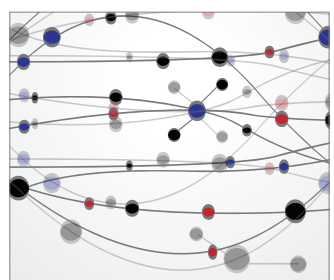

\section{The Scientific} World Journal
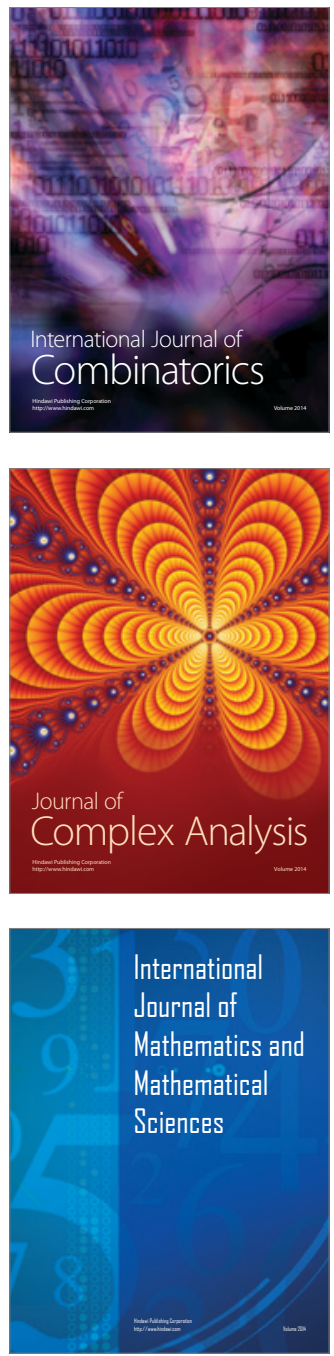
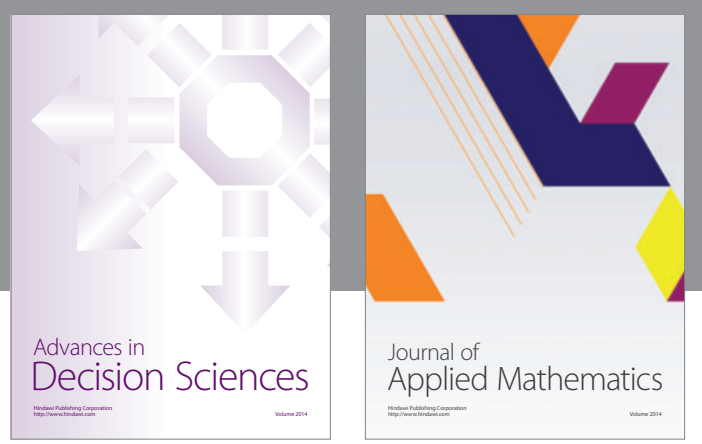

Algebra

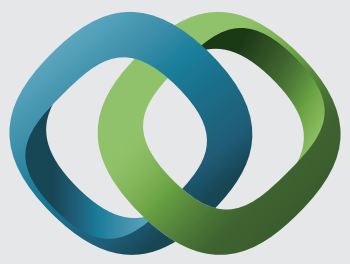

\section{Hindawi}

Submit your manuscripts at

http://www.hindawi.com
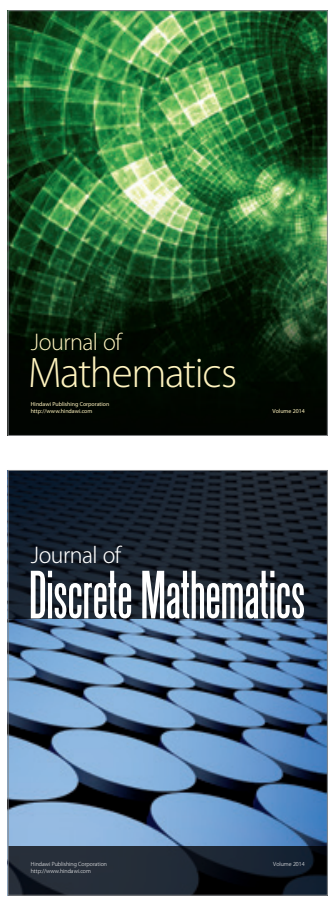

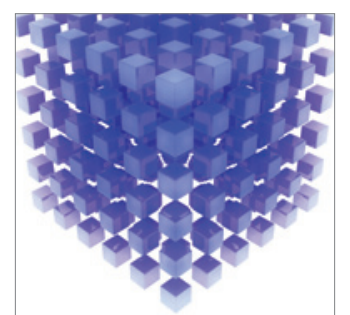

Mathematical Problems in Engineering
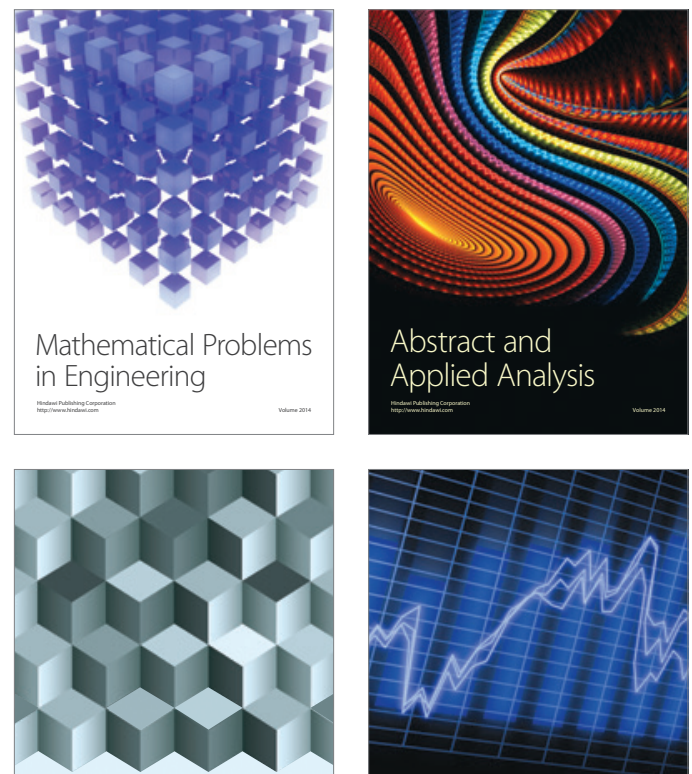

Journal of

Function Spaces

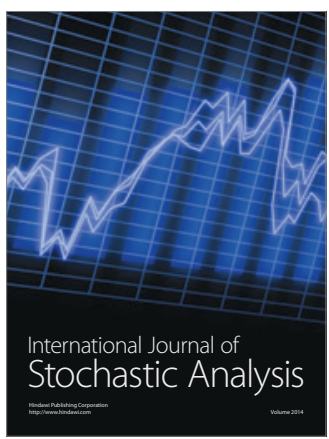

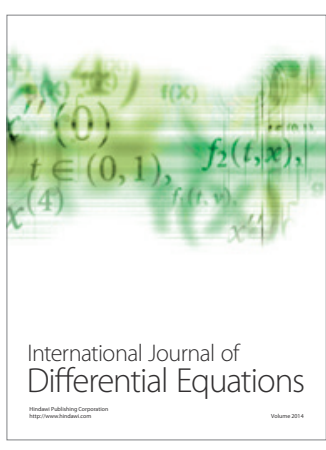
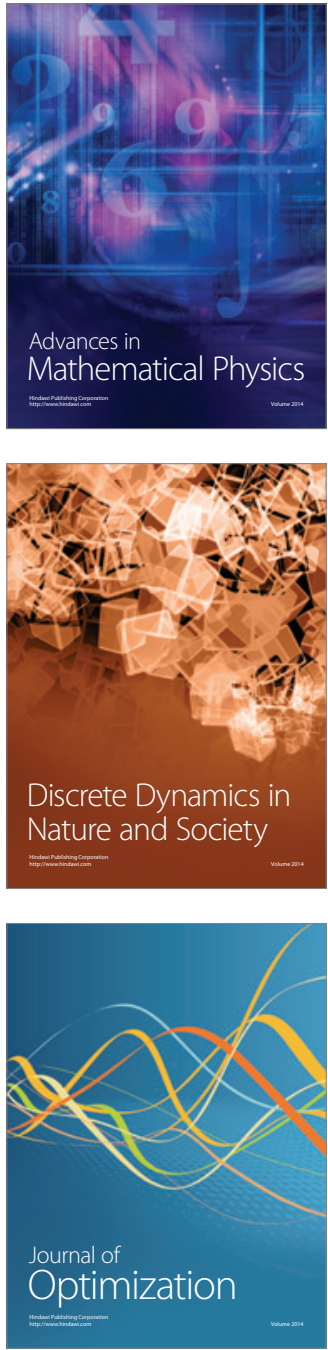